\title{
Design Of Low Power 6t-Sram Cell For Advanced Processors
}

\author{
Sujata A, Lalitha Y.S
}

\begin{abstract}
The Static Random Access Memory (SRAM) is one of the feature of the robotized world. Everything thought of it as, channels creature level of intensity \& bomb wretchedly zone. In that point of confinement wide investigate in the SRAM is an advancing related power dispersal, memory chip zone \& supply voltage major. This paper SRAM assessment to the degree Static Noise Margin, Data Retention Voltage, Read Margin \& Write Margin for low control application is considered. The Static Noise Margin (SNM) is one of the very peak head for essentials of dealing with memory since it effects read edge sensibly as the structure edge. In the SRAM cell SNM is identified with the NMOS \& PMOS contraption's most purged point respects. The High Read \& Write Noise Margin is other than true bugs in the structure of the SRAM information retention Voltage is consented to 6T-SRAM cell for the applications requiring lively works out. The Various sorts of wind are taken unmistakably to examinations to the 6t-SRAM by fluctuating the size of the transistor. The Execution appraisal is examined in 6T-SRAM oversaw and finished in 32nm progression.
\end{abstract}

Keywords: SRAM, 6T-SRAM, Noise Margin, Read boundary, Write Margin, Data Retention Voltage, Virtuoso.

\section{INTRODUCTION}

These days one of the totally used Electrcal contraption or Electronic circuit is Static Random Access Memory (SRAM) [1]. The Quality of the SRAM is produced when it using the CMOS degrees of progress all around depend upon the SNM. SRAM memory advancement is used in light of its speed and sufficiency. The contraption of the diminished in sizes of a few structure disturbs arise in the nanometer size SRAM plan. In the SRAM cell movement everything considered as supply voltage scaling is performed. The base voltage appeared as Data Retention Voltage (DRV) and it is required for a SRAM cell to store the data. An Decreasing the VDD lessens sub-edge spillage Current and Territory spillage. Unquestionably when VDD is diminished too far data hardship occurs in the SRAM. The DRV is connected with shield data in the bit- cells of SRAM. To keeping sharp SRAM estimation of read edge \& structure edge is essential. A mammoth vision in this paper is to study about 6T SRAM cell and it relies upon fomenting effect edges by examining the DRV, read Margin and Write Margin. Present- days spotlight is one of low supply voltage which diminishes the SNM. The enduring idea of the SRAM cell can be annihilated subject to the SNM regard since execution relating to the SNM. So as SNM decreases the introduction of SRAM cell nearly lessens or the a substitute way. To improving the introduction of a 6T SRAM cell parameter, for instance, cell degree (CR), pull up degree (PR), voltage supply (VDD) are routinely considered. The key prelude to 6T SRAM cell building gives in piece I, separate II explains the SRAM working and locale III clarifies the SRAM w.r.t to Static Noise Margin (SNM), Read Margin (RM), Write Margin (WM) and Data Retention Voltage (DRV) in the bit IV SRAM implementation methodology is given and SRAM's Simulation Results are explained in V. In part VI references are given.

\section{STATIC THEORETICAL ACCESS MEMORY (SRAM)}

The most of a wide edge of the pushed structures these days consolidate SRAM. In modernized structures, the thickness of outline \& speed of execution is the most explored part. Contraptions are scaled to as an accomplish less multifaceted nature, supply voltages and edge voltages. To understand 1 search for a Six-Transistor memory cell which is generally observed in the standard memory cell? For accomplish more thickness, the memory cells ought to be diagramed fittingly a standard sixT sram methodology is considered. This SIX T SRAM handles 1V power supply standard $90 \mathrm{~nm}$ for its development when stood detached from $1.8 \mathrm{~V}$ in standard $180 \mathrm{~nm}$. On a off chance that the supply voltage related with the improvement of SRAM is low, by then control use is in like way reduced. The information bits is to be regulated in SRAM are connected with the cross coupled inverters.
Revised Manuscript Received on August 14, 2019.
Sujata A, Research Scholar at Appa Institute of Engineering \&
Technology, Kalaburagi, Karnataka, India (email:
sujata.kamlapurkar@gmail.com)
Dr. Lalitha Y.S, Department of Electronics \& Communication
Engineering, Don Bosco Institute of Technology, Bangalore, Karnataka, India. 


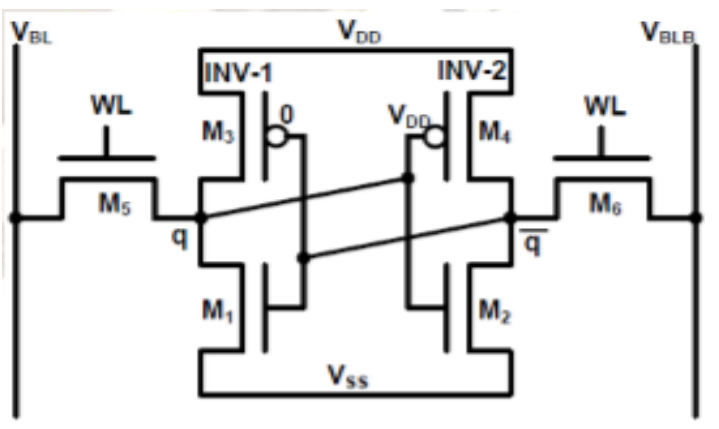

Figure 1: SRAM Schematic

The cross coupled inverters has two stable states '0' \& ' 1 '. These are connected beside to the SRAM .The other two transistors named as transistors and driver transistor. These two transistors are to control the isolated improvement \& make assignments out of 6T SRAM. The SRAM cell is everything seen as related by the word line (WL) controlling M5 and M6. The transistors M5 and M6 control data moving in read and make assignments to improve both edges purposeless lines are routinely given in the SRAM. For the investigation of the level of need sixT SRAM, the speculation of static tumult edge (SNM) is connected [2].

\section{A. SRAM WORKING}

The SRAM everything dismembered works in three frameworks for development, to be a unequivocal hold mode, read mode and structure mode. Unequivocally when SRAM in stronghold mode or Hold mode a Word Line is associated with the ground. The SRAM holds data without flipping the data \& Data is held in the S-RAM till power is connected. When the SRAM is in Read mode, bit-line are pre-charged to the voltage VDD and the word-line is set. To take a gander at the ' 0 ' or ' 1 ' the bit-line is generally discharge through the way transistor. Discharging Current is looked M1 and M5 and it is settled in condition 1. The cell degree, is plot for the level of the drive transistor and stack transistor. The SNM depend upon the Cell Ratio. In this way it is watched that as the phone degree grows SNM of the memory cell extending rates achieve improvement of current in a memory cell [4].

$$
\begin{aligned}
& \beta_{n, M 5}\left\{\left(V_{D D}-V_{Q B}-V_{t n}\right) V_{D S A T n}-\frac{V_{S A T m}}{2}\right\}=\beta_{n, M 1}\left\{\left(V_{D D}-\right.\right. \\
& \left.\left.V_{t n}\right) V_{Q B}-\frac{V_{Q B}^{2}}{2}\right\}
\end{aligned}
$$

The equation 1 is simplified in equation 2

$$
\begin{aligned}
& V_{Q B}=\left\{V_{S S A T n}+C R\left(V_{D D}-V_{t n}\right)-\right. \\
& \left.\sqrt{V_{D S A T n}^{2}(1+C R)+C R^{2}\left(V_{D D}-V_{t n}\right)^{2}}\right\} \div C R
\end{aligned}
$$

Where the Cell Ratio (CR) also known as ? ratio represented in equation 3 .

$$
\text { Cell Ratio }(C R)=\frac{w_{1} / L_{1}}{w_{5} / L_{5}}
$$

The draw up of degree is portrayed as the level of the stack transistor and the way transistor. Therefore it is watched that as the draw up degree swarms SNM of the memory cell increases. The Current experiencing M4 and M6 are kept in condition 4 with accreditation that make is accomplishment [7].

$$
\begin{aligned}
& \beta_{n, m n 6}\left\{\left(V_{D D}-V_{t n}\right) V_{Q}-\frac{V_{Q}^{2}}{2}\right\}=\beta_{p, M 4}\left\{\left(V_{D D}-\right.\right. \\
& \left.\left.V_{t p}\right) V_{D S A T p}-\frac{V_{D S A T p}^{2}}{2}\right\}
\end{aligned}
$$

This equation four is simplified to equation five as

$$
\sqrt{V_{Q}=V_{D D}-V_{t n}-}
$$

Where PR is called Pull up Ratio or ? Ratio and is represented in equation 6 is

$$
\text { Pullup Ratio }(P R)=\frac{w_{4} / L_{4}}{w_{6} / L_{6}}
$$

\section{STATIC NOISE MARGIN}

The SNM is the square- hovered in the crucial essential voltage move characteristics (VTC) and reflected voltage move properties. The estimations of CR nine, PR [11] \& VDD 10 ordinarily recognize gigantic occupations in the appraisal of the Static Noise Margin of a 6T SRAM cell. SNM should be of High the motivation for high plentifulness of the SRAM cell. Figure 2 watches out for a general relationship for a SRAM bit-cell holding data and to address SNM.

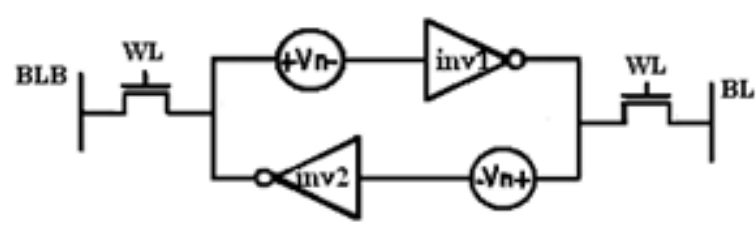

Figure 2: The General setup of SRAM for SNM [2]

In the SRAM cell SNM is likewise subject to look at edge and make edge. For quantify of the SNM Butterfly methodology in 6T SRAM cell is commonly view as where the voltage move qualities turns are pivoted to make butterfly structure 12 .

To process SNM in CMOS the channel current considered as 


$$
\begin{aligned}
& I_{D}=\frac{1}{2} \beta\left(V_{G S}-V_{T}\right)^{2} \\
& I_{D}=\beta V_{D S}\left(V_{G S}-V_{T}-\frac{1}{2} V_{D S}\right)
\end{aligned}
$$

In the saturated and linear regions, SNM is calculated as $S N M_{6 T}=V_{T}-$

$\left(\frac{1}{(k+1)}\right)\left\{\frac{V_{D D}-(2 r+1) /(r+1) V_{T}}{1+\frac{T}{k(r+1)}} \frac{V_{D D}-2 V_{T}}{1+k \frac{r}{q}+\sqrt{\frac{r}{q}}\left(1+2 k+\frac{r}{q} k^{2}\right)}\right\}$

(9)

$$
\begin{aligned}
& r=\text { ratio }=\frac{\beta_{A}}{\beta_{a}} \quad(10) \\
& q=\frac{\beta_{p}}{\beta_{a}} \\
& V_{\mathrm{T}}=\text { Threshold voltage } \\
& k=\left(\frac{r}{r+1}\right)\left(\left\{\sqrt{\frac{r+1}{r+1-\left(V_{S}\right)^{2} /\left(\mathrm{v}_{T}\right)^{2}}}-1\right\}\right) \\
& V_{S}=V_{D D}-V_{T} \\
& V_{r}=V_{S}-\left(\frac{r}{r+1}\right) V_{T}
\end{aligned}
$$

From the graphical analysis the Voltage Transfer Characteristic of the Inverter 2 and the inverse VTC 1 from Inverter 1 is plotted and the two lobed curves formed as "butterfly curve" and considered for the analysis of the SNM in the SRAM.

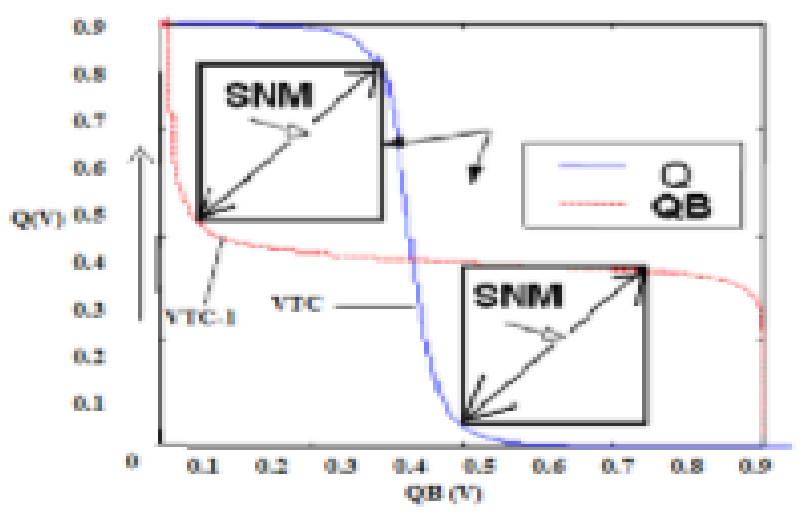

Figure 3: Calculation of SNM

From the Figure 3 the SNM can be considered as the side of the two squares kept between the two VTCs of a SRAM cell the voltage move trademark of one cell inverter superposes the voltage move trademark of the other cell inverter 13. The two-lobed graph shaped is consistently known as a "butterfly" bends and is bankrupt down to pick the SNM of the SRAM. The SNM from this strategy regarding to bend tended and it is depicted as the side length of the best square which can be fitted inside the projections of the "butterfly" wind.

\section{A. Read Noise Margin}

The cell holds its state in read activity. During read activity the phone_ is unprotected if the Read-SNM diminishes. The explanation behind the powerlessness is that when discovering_ Read-SNM, pre-charging the wordline and bit-line to the high- respect occurs due to the voltage -segregating impact over the path transistor and drive transistor internal focus purpose of the bit-cell tending to a "o" gets pulled upward through the way transistor in this way demolishing in SNM during the read development happens. Therefore an off kilter information might be dealt with in the cell due to the change in its state in a read cycle. Figure four shows the VTC curve for RSNM properties \& subject to this contort Read edge is settled. The read edge depicts the read power of the "SRAM" cell. In the SRAM cell the information support fortification mode and read access and it is a basic control. The SRAM cell is an stability reduces with the supply voltage decay thusly developing the spillage current happening because of scaling in the advancement. On the off chance estimation of the SNM is expanded \& the read dependability of the SRAM cell increments. The SRAM* cell with high" RSNM" has mind blowing analyzed quality.

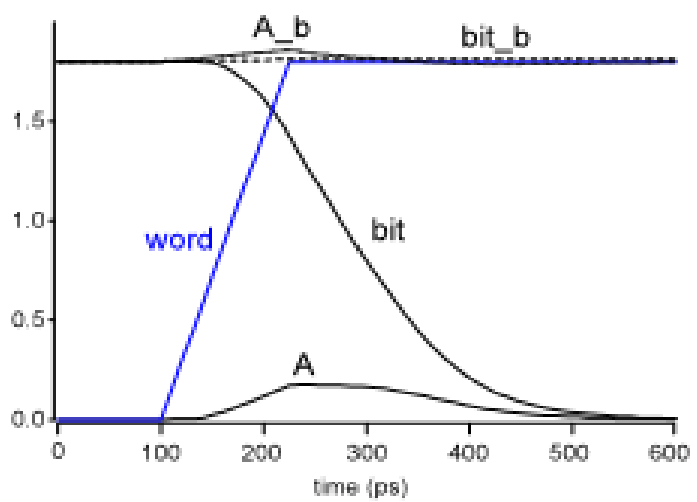

Figure 4:Read Margin from SNM.

By proposing the Figure Three( 3) the _SNM can be considered as the side of the two squares kept between the two "VTCs " of a *SRAM cell the voltage move trademark of one cell inverter superposes the "voltage move trademark " of the other cell inverter 13. The two-lobed outline confined is reliably known as a "butterfly" bend \& is bankrupt down to pick the ${ }^{\wedge} \mathrm{SNM}^{\wedge}$ of the -SRAM. The *SNM from this technique with respect to twist tended to is depicted as the side length of the best square which can be fitted inside the projections of the 'butterfly' wind.

\section{B. Read Noise Margin}

"The cell holds its state in a read advancement" during read advancement the telephone is unprotected if the ReadSNM diminishes. A clarification behind weakness is that when finding Read_SNM, pre-charging the word_line \& bit_line to a high regard occurs due to the voltage binding effect over the way transistor and drive transistor internal center reason behind the 'bit-cell' keeping an eye out for a zero gets pulled upward through the course transistor along these lines crushing in $\sim$ SNM read improvement occurs. In this way an inconsistent data may be directed in the cell due to change in its state in a read cycle. Figure four shows $\sim$ VTC wind for $\sim$ RSNM properties and subject to this bend Read- edge is settled. The" read edge" portrays the read power of the _SRAM cell. In the *SRAM cell the data support in fortress mode and read access is a pivotal control. The /SRAM/ cell stability reduces with the supply voltage rot as such stirring up the spillage current happening as a result of scaling in the development. If the estimation of ${ }^{*} \mathrm{SNM} *$ is widened, the read steadfast nature of the *SRAM cell increases. Along these lines a $<\mathrm{SRAM}>$ cell with high $<$ RSNM $>$ has mind blowing examined quality.

The SNM as a criterion is the most common approach. If the WSNM is minimized the write ability decreases. 


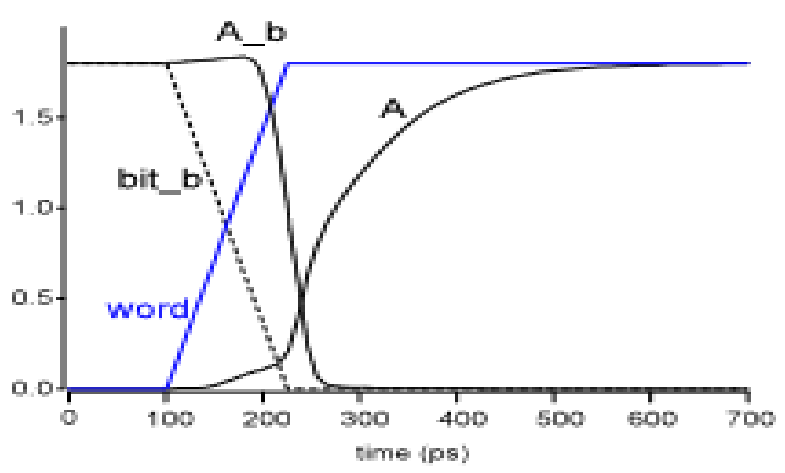

Figure. 5:WriteMargin Calculation from< SNM $>$.

\section{Data Retention Voltage ( $* D R V *)$}

Data Retention Voltage is the base VDDnecessary for holding the information for the ${ }^{\wedge}$ SRAM Cell [15]. Two center centers $(\mathrm{Q}$ and $\mathrm{Qb})$ are open in !SRAM cellto store values of ' 0 ' or ' 1 ' [14]. When decreasing the $\sim$ VDD the data in the -SRAM cell remain immovable and at a particular voltage flip in the area of $\sim$ SRAM cell occurs, the voltage at which flipping happens the Data Retention Voltage is grabbed.

"Inside inverters Voltage Transfer Curves "contaminates to a level that /SNM of the ${ }^{\wedge} \mathrm{SRAM}$ cell reduces to zero as shown up in the figure 6 . In the case of the \{SRAM \}cell $\mathrm{q}={ }^{\prime} 1$ ', $\mathrm{qb}=\mathrm{C}^{\prime} 0$, it flips the value toq='0', qb='1'. The power supply voltage [VDD] value diminishes, thus data upkeep voltage should be truly more than the farthest point voltage. The breaking point voltage is $200 \mathrm{mV}$, thereby *SRAM* flips its state of the value and diminished underneath 200 $\mathrm{mV}$ open for later/read mode.
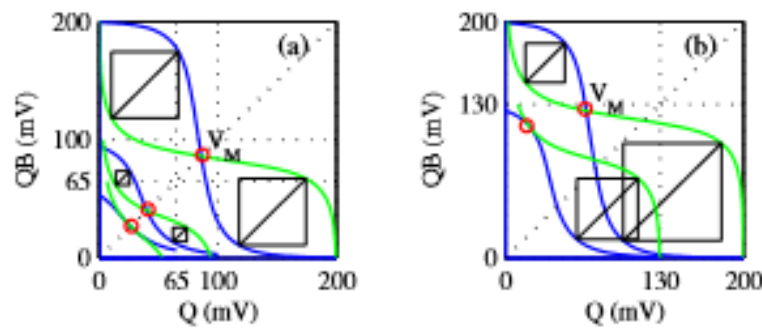

Figure6: <VTC > of |SRAM | cell during LDRV calculation

\section{IMPLEMENTATION METHODOLOGY \& RESULTS}

The 6T ISRAM $\backslash$ is executed in rhythm fundamental suite in $32 \mathrm{~nm}$ movement and it would be a rule in figure 7 . Everything considered all through movement the size of *NMOS* and /PMOS/ transistors is $100 \mathrm{~nm}$. The discover of the cell degree transistor NM2 is considered as driver transistor and NM3 is considered as the stack transistor. The degree of the driver transistor to stack transistor considered is in the degree of 1 to 2.5 for read improvement. Subsequently the RM is veering from the CR to discover the pull-up degree transistor PM1 is considered as weight transistor and NM4 is considered as the way transistor. The degree between access transistor and load transistor considered is in the degree of 3 to 4 . Thusly the make edge is truly concerning the pull-up degree. The SNM is genuinely looking voltage VTh and the $\langle\mathrm{DRV}\rangle$ is plainly relative purpose of control voltage VTh for $32 \mathrm{~nm}$ development the edge voltage of $200 \mathrm{mV}$ is commonly considered.

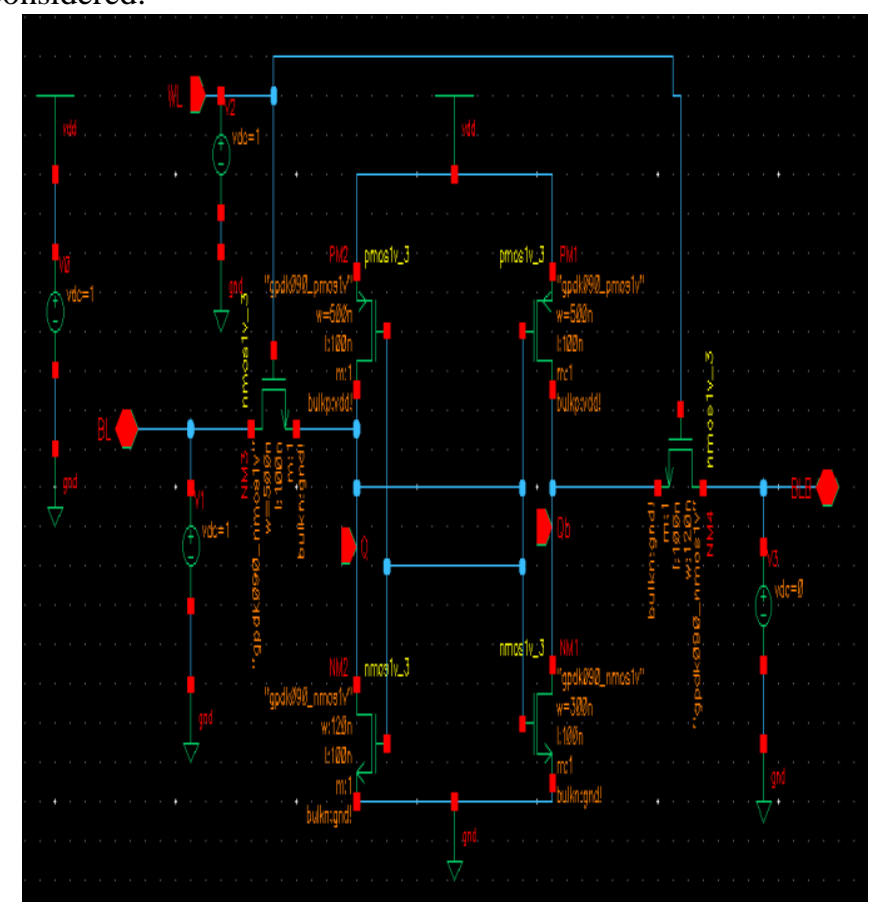

Figure 7: Schematic diagram of SRAM Cell

\section{SIMULATION RESULTS}

The dependency of SNM with respect to the Cell Ratio (CR) is tabulated in Table 1 and the SNM for Cell Ratio from 0.8 to 1.6 is plotted in figure 8 (a) to 8 (e).

Table 1: CR vs SNM

\begin{tabular}{|c|c|c|c|c|}
\hline $\begin{array}{c}\text { Cell } \\
\text { Ratio }\end{array}$ & $\mathrm{W}_{1}$ & $\mathrm{~W}_{3}$ & $\mathrm{~W}_{3}$ & SNM \\
\hline 0.8 & 1.6 & 2.0 & 6.4 & $394.9-134.2=260.7$ \\
& $\mu \mathrm{m}$ & $\mu \mathrm{m}$ & $\mu \mathrm{m}$ & \\
\hline 1.0 & 2.0 & 2.0 & 8.0 & $326.3-62.7=263.6$ \\
& $\mu \mathrm{m}$ & $\mu \mathrm{m}$ & $\mu \mathrm{m}$ & \\
\hline 1.2 & 2.4 & 2.0 & 9.6 & $352.3-61.1=291.2$ \\
& $\mu \mathrm{m}$ & $\mu \mathrm{m}$ & $\mu \mathrm{m}$ & \\
\hline 1.4 & 2.8 & 2.0 & 11.2 & $402.4-59.27=343.1$ \\
& $\mu \mathrm{m}$ & $\mu \mathrm{m}$ & $\mu \mathrm{m}$ & 3 \\
\hline 1.6 & 3.4 & 2.0 & 12.8 & $410.8-49.61=361.1$ \\
& $\mu \mathrm{m}$ & $\mu \mathrm{m}$ & $\mu \mathrm{m}$ & 9 \\
\hline
\end{tabular}

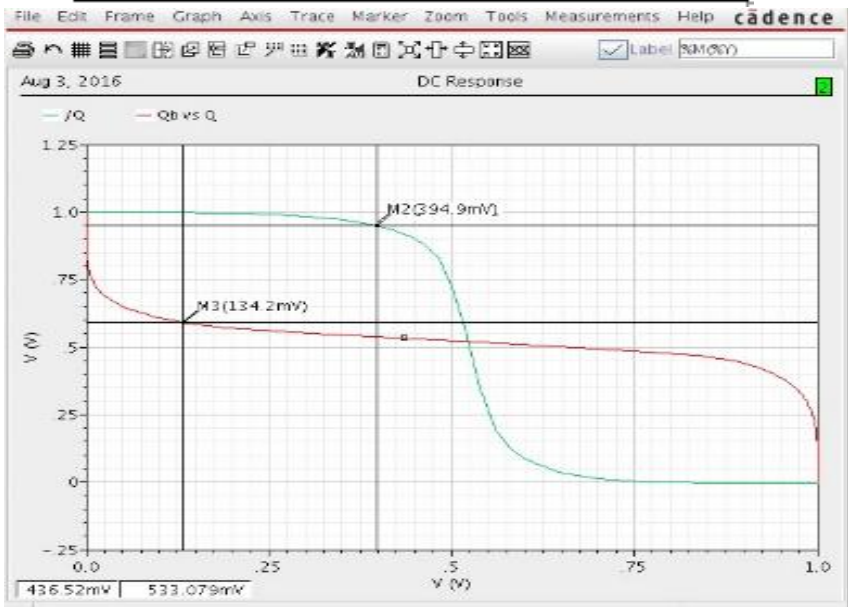

(a) 


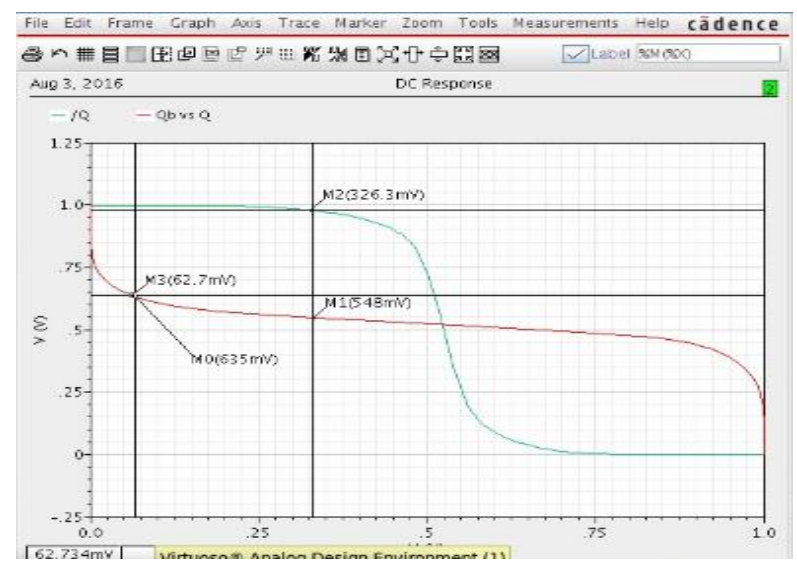

(b)

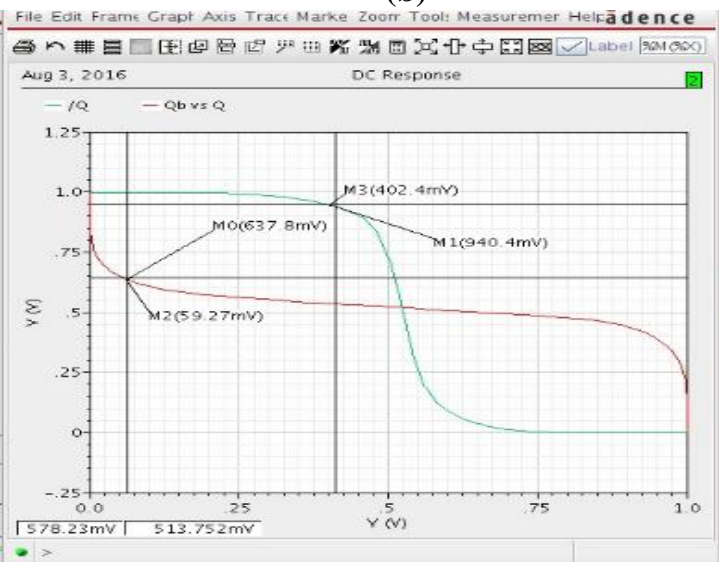

(c)

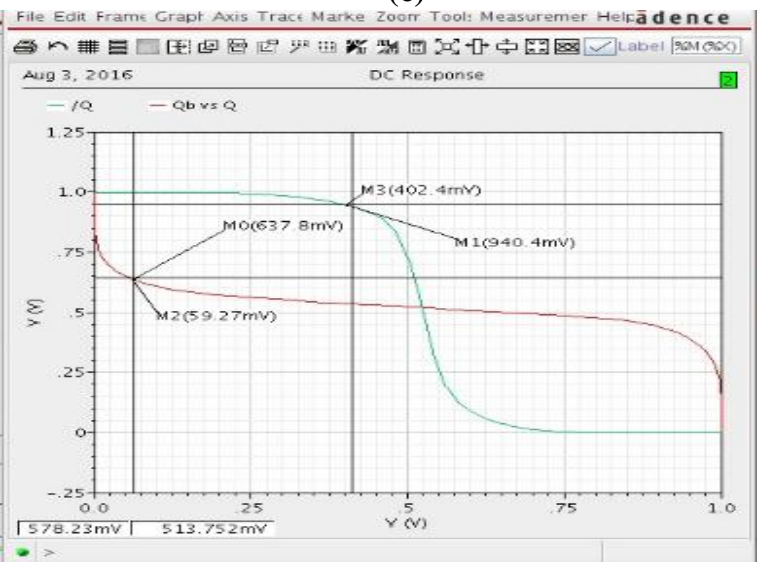

(d)

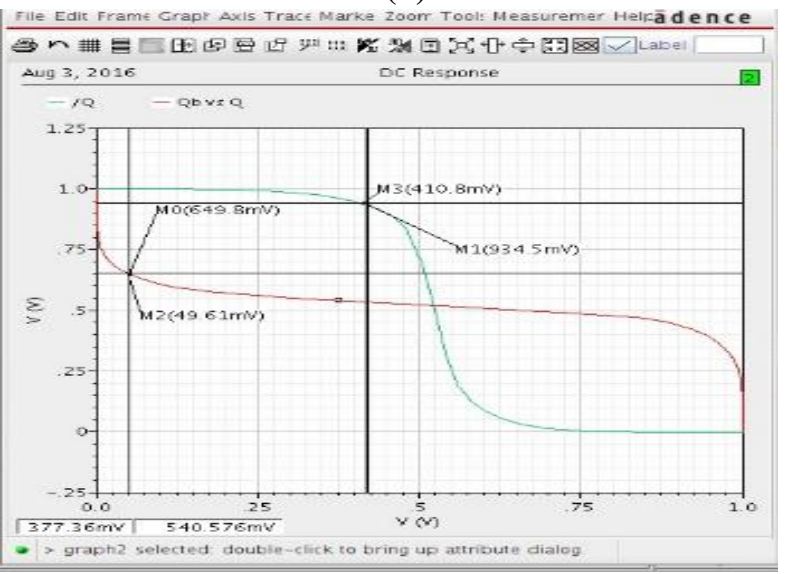

(e)

Figure 8: (a) $\mathrm{SNM}$ vs $\mathrm{CR}$ when $\mathrm{CR}=0.8(\mathrm{~b}) \mathrm{SNM}$ vs $\mathrm{CR}$ when $C R=1.0$ (c) $\mathrm{SNM}$ vs $\mathrm{CR}$ when $\mathrm{CR}=1.2$ (d) $\mathrm{SNM}$ vs

$C R$ when $C R=1.4$ (e) $S N M$ vs $C R$ when $C R=1.6$
With the increase in cell ratio, SNM also increase, but the trade-off is the size of the transistors. For example to get SNM as $263.7 \mathrm{mV}$ the cell ratio is just 0.8 , it means that it requires less area. Whereas when the SNM is $361.19 \mathrm{mV}$ the required cell ratio is 1.6 which is twice the area for $263.7 \mathrm{mV}$ SNM. Hence it is inferred from figure 9 that to get better SNM, areas has to be sacrificed.

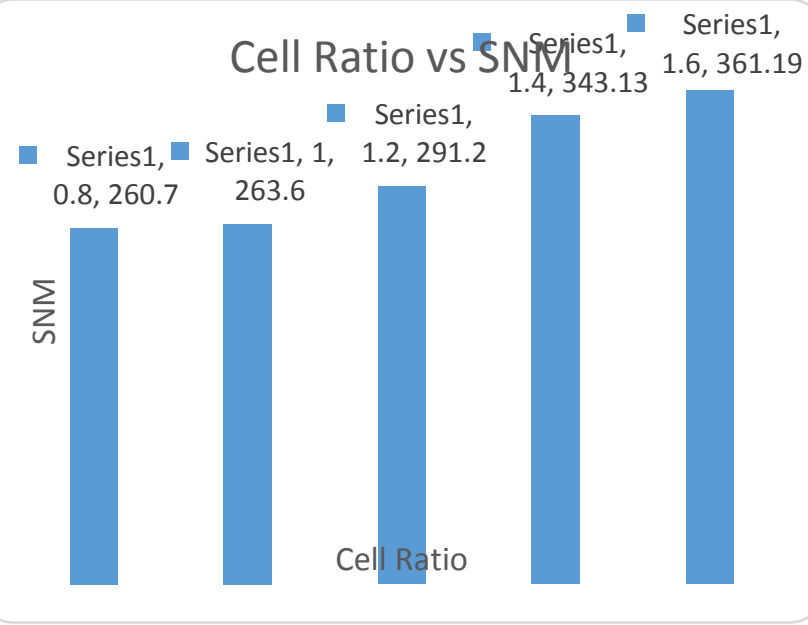

Figure 9: Graph of SNM vs CR

The dependency of SNM with respect to the Data Retention Voltage (DRV) is tabulated in Table 2 and the SNM for DRV from 1.0 to 0.2 is plotted in figure 10 (a) to 10 (e).

\begin{tabular}{|l|l|}
\multicolumn{1}{c}{ Table 2: DRV vs SNM } \\
\hline DRV & SNM \\
\hline 1.0 & $362.5-67.76=294.74$ \\
\hline 0.8 & $337.0-47.44=289.56$ \\
\hline 0.6 & $238.8-13.54=225.26$ \\
\hline 0.4 & $159.9-16.74=143.16$ \\
\hline 0.2 & $85.03-40.15=44.88$ \\
\hline
\end{tabular}

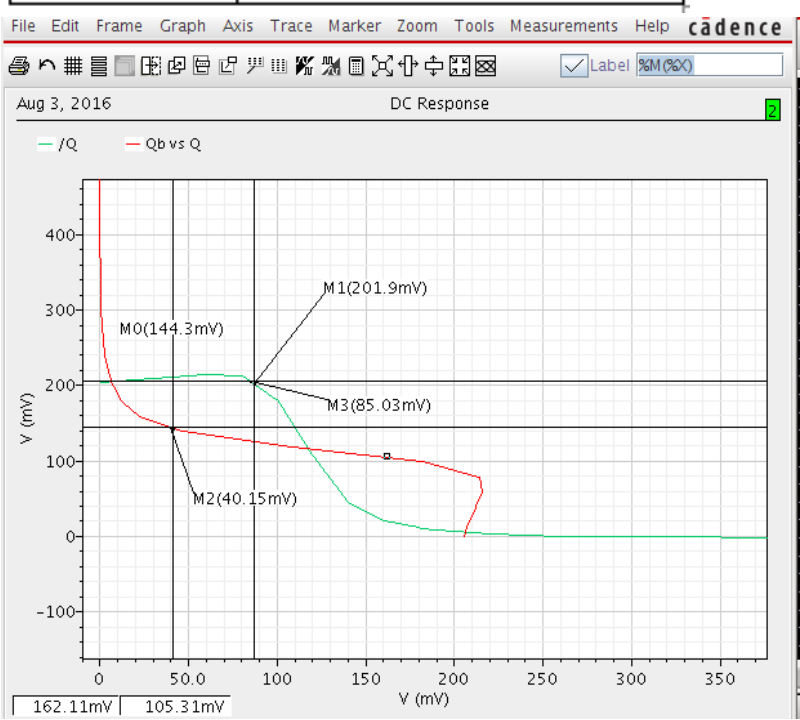

(a)

Published By: 


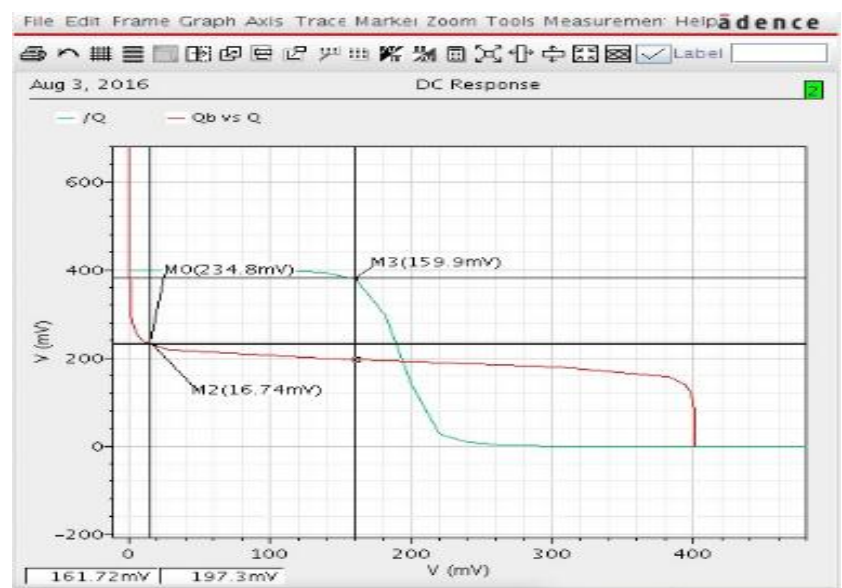

(b)

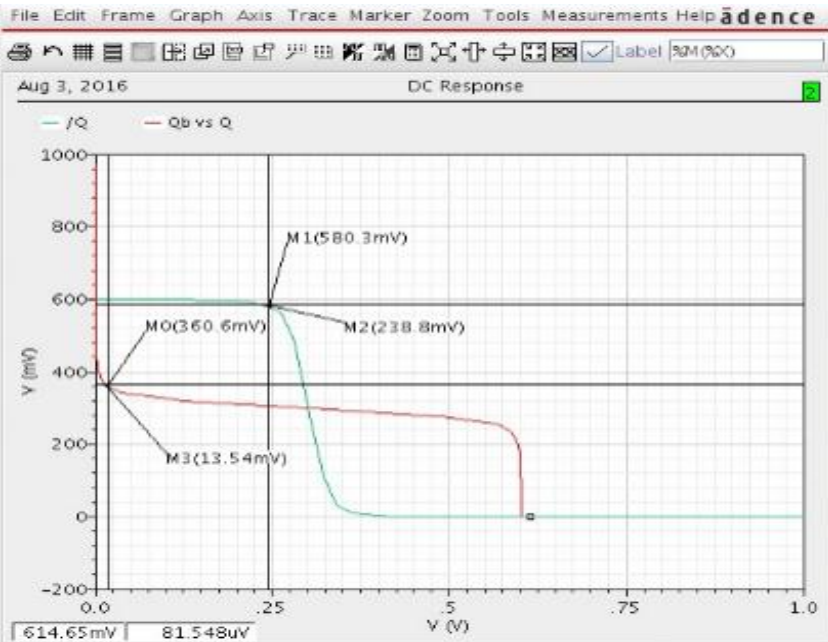

(c)

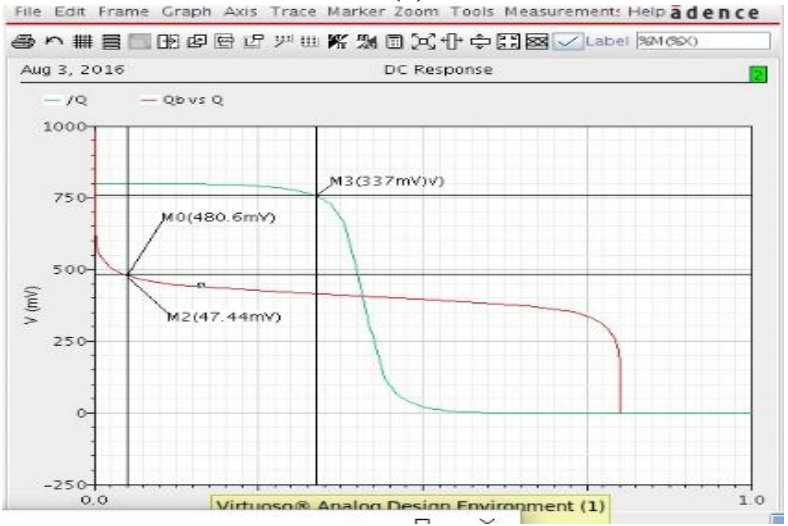

(d)

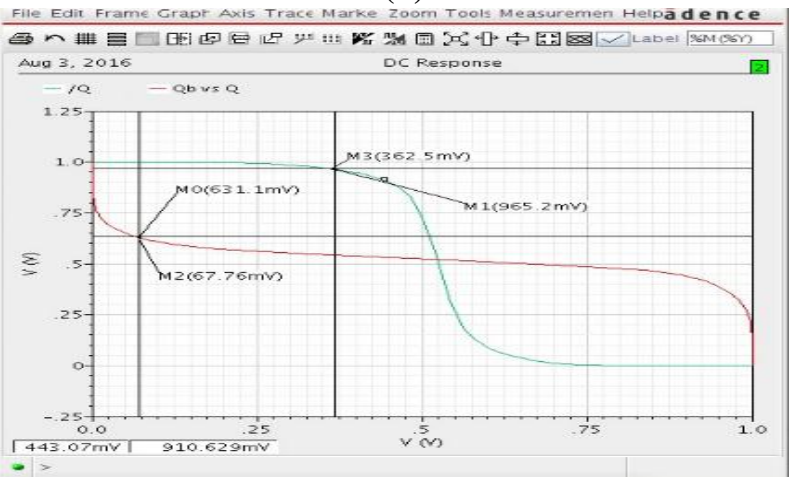

(e)

Figure 10: (a) SNM vs DRV when DRV=0.2(b) SNM vs $D R V$ when $D R V=0.4(c) S N M$ vsDRV when $D R V=0.6$

(d) SNM vs DRV when DRV=0.8 (e) SNM vs DRV when DRV=1.0
For DRV analysis normal VDD $=1 \mathrm{~V}$ for $32 \mathrm{~nm}$. The

VDD is reducetill the data is flipped. The data is flipped almost at $200 \mathrm{mV}$ because the Vth of the transistors in $32 \mathrm{~nm}$ technology is $180 \mathrm{mV}$. DRV can't be less than the Vth. For $32 \mathrm{~nm}$ technology SRAM DRV $=200 \mathrm{mV}$ which should be more than the Vth $(180 \mathrm{mV})$ Assuming Cell Ratio $=1$ and setting width of transistor M1 and M5 to be $2.0 \mu \mathrm{m}$

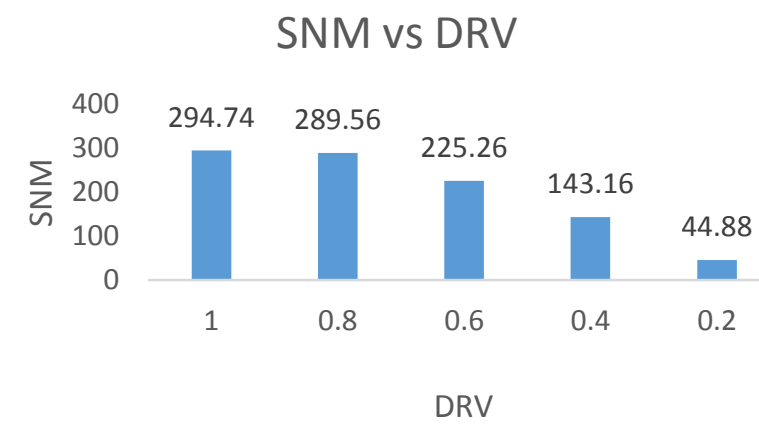

Figure 11: Graph of SNM vs DRV

The dependency of SNM with respect to the Cell Ratio is calculated to find the respective Read Marginwhich is tabulated in Table 3 and the SNM for Cell Ratio from 0.8 to 1.6 is plotted in figure 12 (a) to 12 (e).

Table3: Read Margin vs SNM

\begin{tabular}{|c|c|c|c|c|}
\hline $\begin{array}{c}\text { Cell } \\
\text { Ratio }\end{array}$ & $\mathrm{W}_{1}$ & $\mathrm{~W}_{3}$ & $\mathrm{~W}_{3}$ & SNM \\
\hline 0.8 & 1.6 & 2.0 & 6.4 & $394.9-134.2=260.7$ \\
& $\mu \mathrm{m}$ & $\mu \mathrm{m}$ & $\mu \mathrm{m}$ & \\
\hline 1.0 & 2.0 & 2.0 & 8.0 & $326.3-62.7=263.6$ \\
& $\mu \mathrm{m}$ & $\mu \mathrm{m}$ & $\mu \mathrm{m}$ & \\
\hline 1.2 & 2.4 & 2.0 & 9.6 & $352.3-61.1=291.2$ \\
& $\mu \mathrm{m}$ & $\mu \mathrm{m}$ & $\mu \mathrm{m}$ & \\
\hline 1.4 & 2.8 & 2.0 & 11.2 & $402.4-59.27=343.1$ \\
& $\mu \mathrm{m}$ & $\mu \mathrm{m}$ & $\mu \mathrm{m}$ & 3 \\
\hline 1.6 & 3.4 & 2.0 & 12.8 & $410.8-49.61=361.1$ \\
& $\mu \mathrm{m}$ & $\mu \mathrm{m}$ & $\mu \mathrm{m}$ & 9 \\
\hline
\end{tabular}

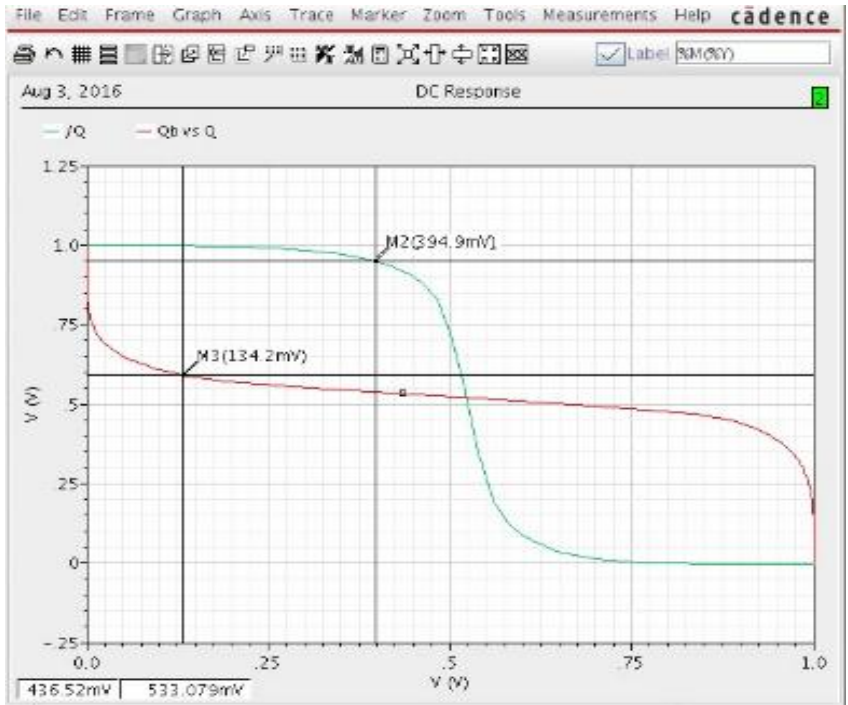

(a)

Published By:

Blue Eyes Intelligence Engineering 


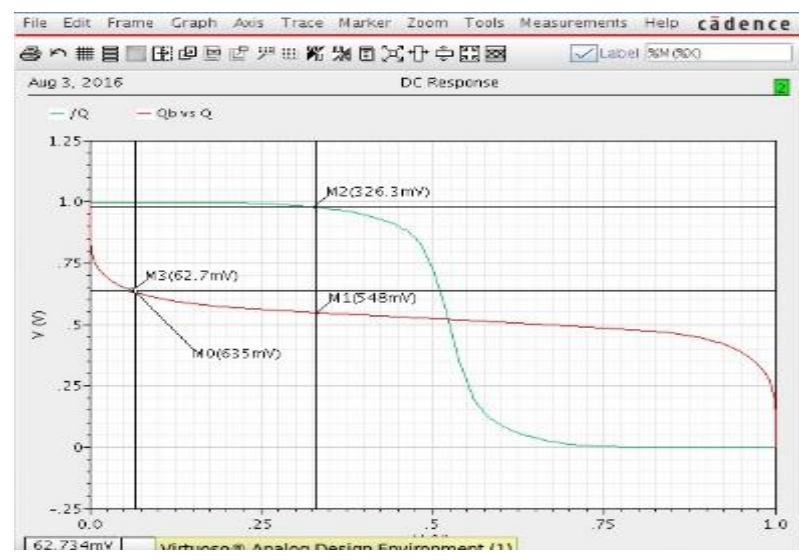

(b)

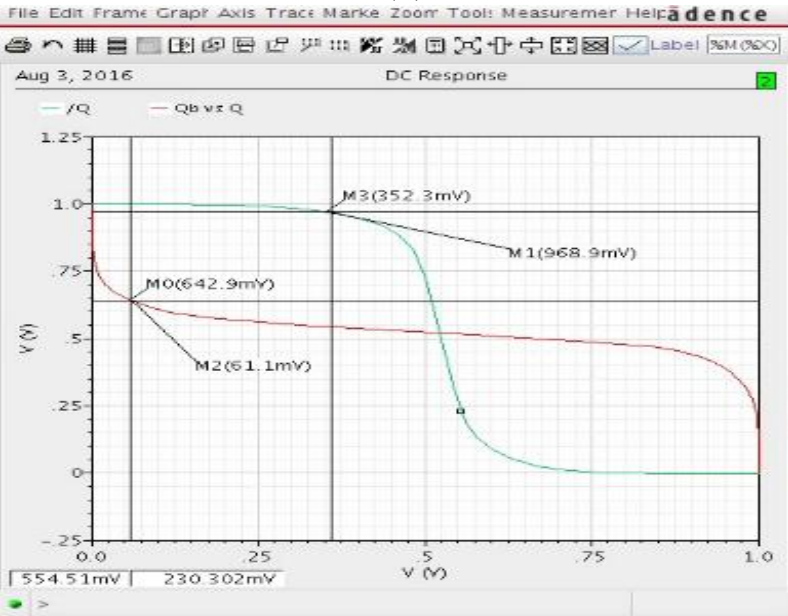

(c)

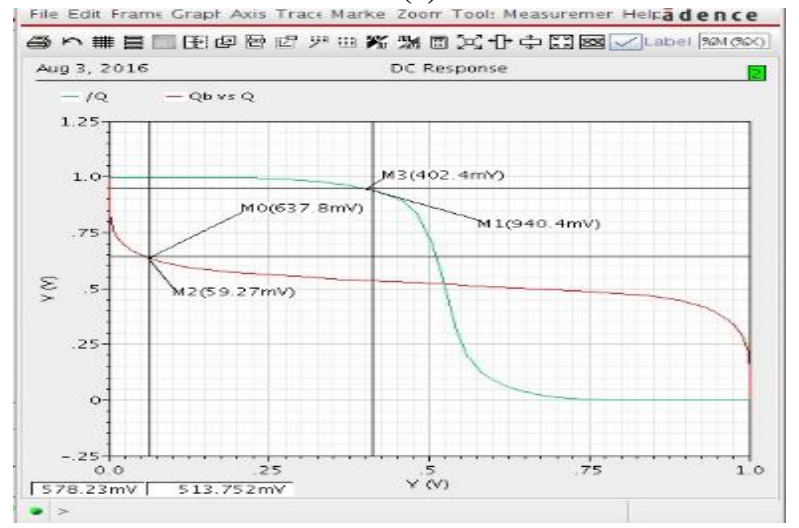

(d)

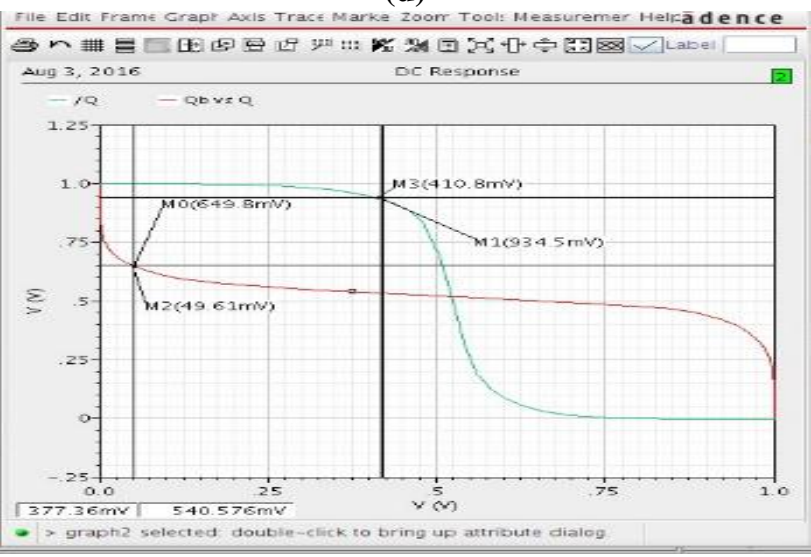

(e)

Figure 12: (a) RMvs SNM when $\mathrm{CR}=\mathbf{0 . 8}(\mathrm{b}) \mathrm{RMvs}$

SNM when $\mathrm{CR}=1.0$ (c) $\mathrm{RMvs} \mathrm{SNM}$ when $\mathrm{CR}=1.2$

(d) $\mathrm{RM}$ vs $\mathrm{SNM}$ when $\mathrm{CR}=1.4$ (e) $\mathrm{RM}$ vs $\mathrm{SNM}$ when $\mathrm{CR}=1.6$
The read margin is calculated as the ratio of SNM to the maximum value while finding SNM. For example, the read margin for the case $\mathrm{CR}=0.8$ is calculated as follows.

Read margin $=260.7 / 394.9=0.660$

\section{Read Margin vs Cell Ratio}

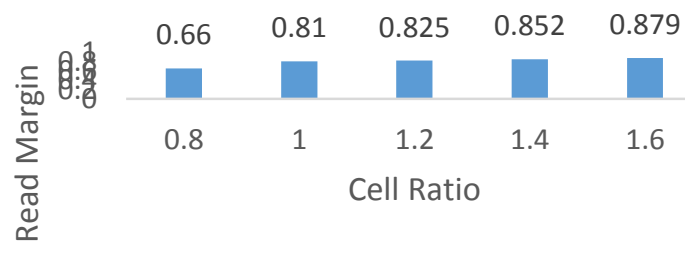

Figure 13: Graph of Read MarginvsCell Ratio

The dependency of SNM with respect to the Pullup Ratio is calculated to find the respective Write Marginwhich is tabulated in Table 4 and the SNM for Pullup Ratio from 3.0 to 4.0 is plotted in figure 14 (a) to 14 (f).

Table 4: Write Margin vs SNM

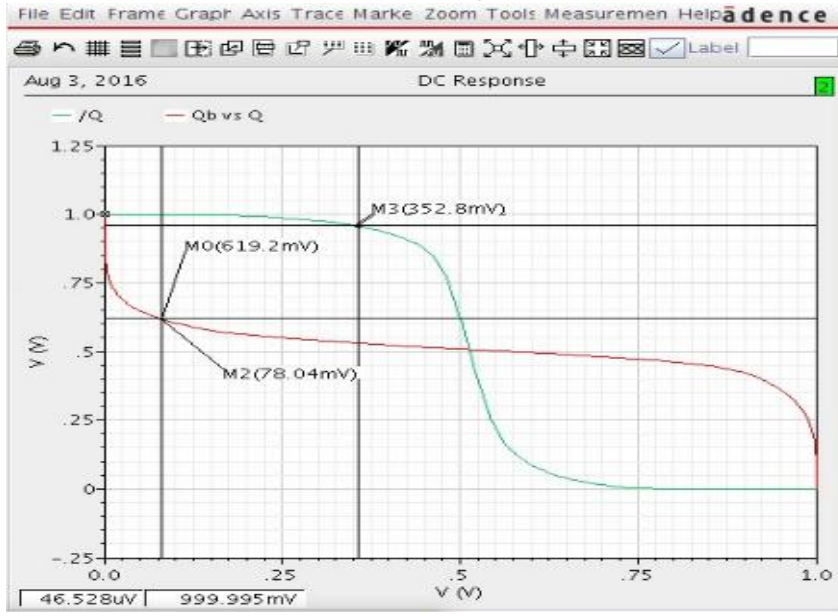

(a)

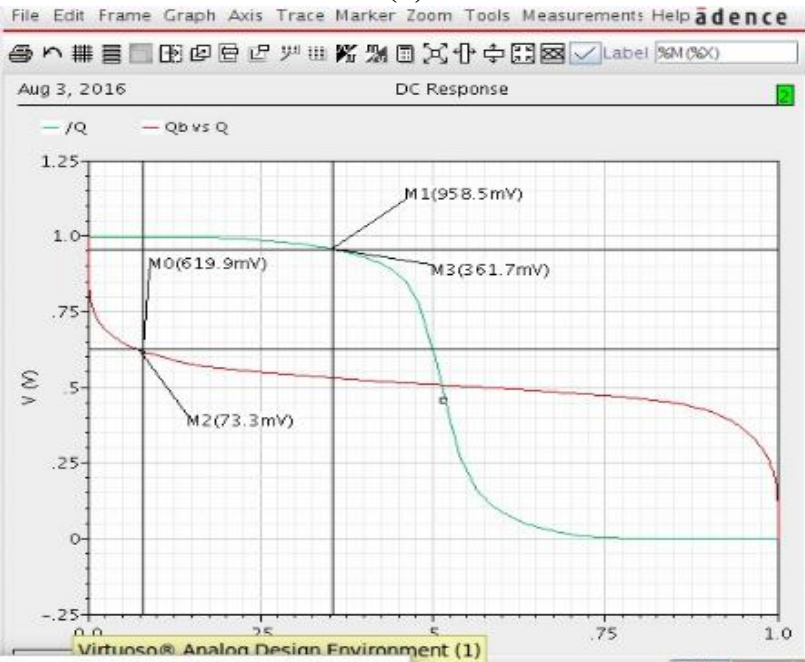

(b)

Published By:

Blue Eyes Intelligence Engineering

\& Sciences Publication 


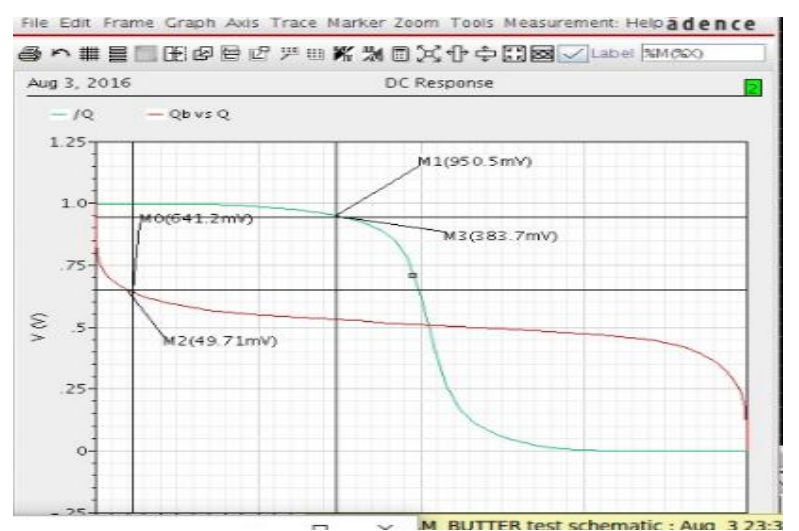

(c)

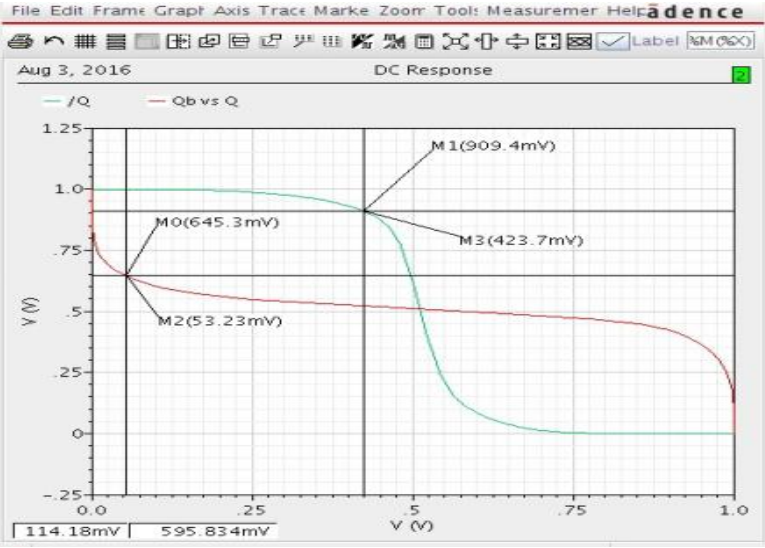

(d)

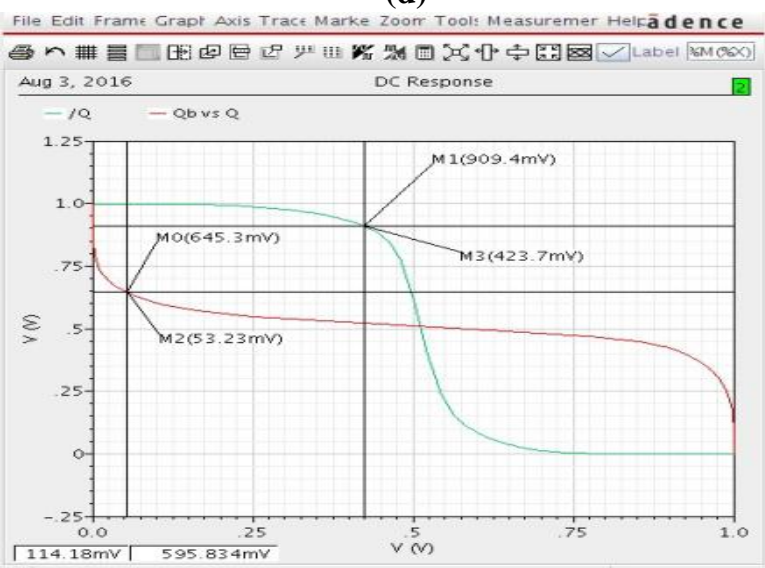

(e)

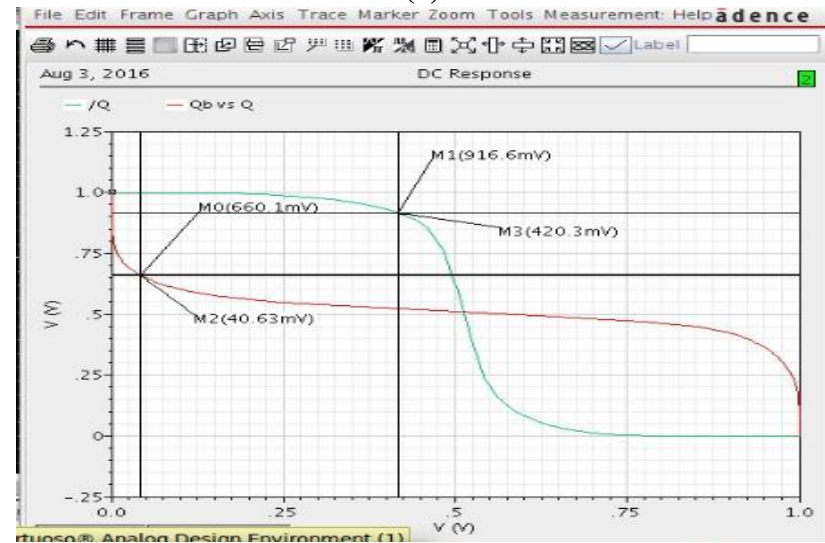

(f)

Figure 14: (a) WMvs SNM when $P R=3.0(b)$ WMvs SNM when $\mathrm{PR}=3.2$ (c) $\mathrm{WMvs} \mathrm{SNM}$ when $\mathrm{PR}=3.4$

(d) WMvs SNM when PR=3.6 (e) WMvs SNM when $P R=3.8$ (f) $W M v s ~ S N M$ when $P R=4.0$
As the pull-up ratio is increased, the SNM is also increased. For example when the PR is 3, the SNM is $274.7 \mathrm{mV}$ whereas the SNM is $379.67 \mathrm{mV}$ when the PR is 4 . It is evident that better SNM can be achieved by sacrificing the area of the transistors. The calculated write margin is specified as

Write Margin $=379.67 / 420.3=0.903$

\section{Write Margin vs Pullup Ratio}

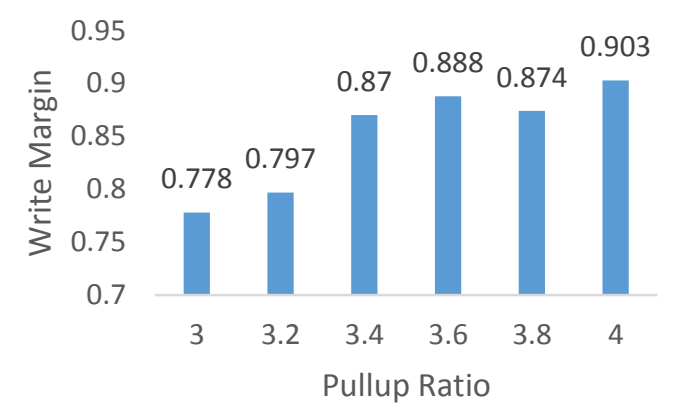

Figure 15: Graph of Write Margin vs Pullup ratio

\section{CONCLUSION}

The $\sim 6 \mathrm{~T}{ }^{\wedge}$ SRAM is composed utilizing ${ }^{\wedge} \mathrm{CMOS}$ transistor and acknowledged in the* CADENCE plan suite in $32 \mathrm{~nm}$ progression. The SRAM was destitute some place around copying it for the parameters like SNM. From the SNM insistence and evaluation is performed on read edge and make edge. The * RM and *WM are checked by considering the estimations of the cell degree of 1 to 2.5 and wreck up degree of 3 to 4 . In light of the execution assessment of relationship among $\sim \mathrm{DRV}$ and $\sim \mathrm{SNM}$ is a statistical model of proposed to survey the 'DRV respect for a $\sim$ SRAM of given size. The gadget estimation is reducing as the progression makes acknowledging variety of $\sim$ VTH which impacts /SRAM cell reliability to unbelievable degree. From the duplication results extraordinary $\{\mathrm{SNM}\}$ is gotten by scarifying the area. The DRV of $0.6 \mathrm{~V}$ is obtained for cell degree of 1 by setting the width of transistors M1 and M5 same. Investigate edge is settled as for SNM is 0.66 and make edge concerning 0.902

\section{REFERENCES}

1. Debasis Mukherjee, Hemanta Kr. Mondal and B.V.R Reddy, Static Noise Margin Analysis of SRAM Cell for High Speed Application, International Journal of Computer Science. 2010 September, 7(5), p.175-180.

2. J. Lohstroh, E. Seevinck, J. de Groot, Worst-case static noise margin criteria for logic circuits and their mathematical equivalence, IEEE J. Solid-State Circuits, 1983 December, 18(6), p.803-807.

3. Ajay Gadhe, UjwalShirode, Read stability and Write ability analysis of different SRAM cell structures, International Journal of Engineering Research and Applications, 2013 February, 3(1), p. 1073-1078.

4. RajshekharKeerthi, Henry Chen, Stability and Static 
Noise margin analysis of low power SRAM, IEEE International Instrumentation \& Measurement Technology Conference, 2008 May, p. 1541-1544.

5. K. Anami, M. Yoshimoto, H. Shinohara, Y. Hirata, and T. Nakano, Design considerations of a static memory cell, IEEE Journal of Solid-State Circuits, 1983 August, 18(4), p. 414-418.

6. Rajni Sharma 1, Sanjay Chopade, Stability Analysis of 6T SRAM at $32 \mathrm{Nm}$ Technology, International Journal of Innovative Research in Science, Engineering and Technology, 2014 May, 3(5), p.12949-12957.

7. AbhishekAgal, Pardeep, BalKrishan, 6T SRAM Cell: Design And Analysis, International Journal of Engineering Research and Applications, 2014 March, 4(3), p. 574-577.

8. GouravArora, Poonam, Anurag Singh,"SNM Analysis of Sram Cells at $45 \mathrm{~nm}, \quad 32 \mathrm{~nm}$ and $22 \mathrm{~nm}$ Technology",International Journal of Engineering Research and General Science Volume 2, Issue 4, pp 785-791.

9. K.Dhanumjaya,Dr.MN.GiriPrasad,Dr.K.Padmaraju,Dr.M .Raja Reddy, Design of Low Power SRAM in $45 \mathrm{~nm}$ CMOS Technology, International Journal of Engineering Research and Applications, 3(4), p. 2040-2045.

10. Seevinck, F. List, and J. Lohstroh, "Static-noise margin analysis of MOS SRAM cells," IEEE J. Solid-State Circuits,Vol. SC-22, pp. 748-754, Oct. 1987.

11. Archnabai, Sram Cell Modeling for Read Stability and Write Ability, International Journal of Emerging Technologies in Computational and Applied Sciences, 2012, p. 26-31.

12. Changhwan Shin, Min Hee Cho, YasumasaTsukmoto, Bich-Yen Nguyen, Carlos Mazure, BorivojeNikolic, TsuJae King Liu "Performance and Area Scaling Benefits of FD-SOI Technology for 6-T SRAM Cells at the 22-nm Node", IEEE Transactions on electron devices, Vol 57, No 6, June 2010

13. A. P. Chandrakasan el al., Low-power CMOS digital design, IEEE Journal of Solid-state Circuits, 1992 April. Volume 2, p. 473-484.

14. AnuragDandotiya, Amit S. Rajput, SNM Analysis of 6T SRAM at $32 \mathrm{NM}$ and $45 \mathrm{NM}$ Technique, International Journal of Computer Applications, 2014 July, 98(7), p. 30-34.

15. Andrei Pavlov \&ManojSachdev, "CMOS SRAM Circuit Design and Parametric Test in Nano-Scaled Technologies". Intel Corporation, University of Waterloo, 2008 Springer Science and Business Media B.V., pp:1-202.

16. Geethumol T.S1,Sreekala K.S, read stability analysis of 6T SRAM bit cell, International Journal of Recent Trends in Engineering and Research, 2013, May, 2(5), p. 155-165.

17. NahidRahman, B. P. Singh, Static-Noise-Margin Analysis of Conventional 6T SRAM Cell at $45 \mathrm{~nm}$ Technology,International Journal of Computer Applications, 2013 March,66(20), p.19-23.

18. NahidRahman,B. P. Singh, Static-Noise-Margin Analysis of Conventional 6T SRAM Cell at 45nm Technology, International Journal of Computer Applications, 2013 March, 66(20).

19. ShivaniYadav, Neha Malik, Ashutosh Gupta and Sachin Rajput, Low Power SRAM Design with Reduced Read/Write Time, International Journal of Information and Computation Technology, 2013, 3(3) (2013), p. 195200.

\section{AUTHORS PROFILE}

Dr. Padmaja Pulicherla, Sujata A Research Scholar at Appa Institute of Engineering \& Technology, Kalaburagi, Karnataka,India

Email: sujata.kamlapurkar@gmail.com
Dr. Lalitha Y.S Department of Electronics \& Communication Engineering, Don Bosco Institute of Technology, Bangalore, Karnataka, India. 